MATEC Web of Conferences 11,02005 (2014)

DOI: $10.1051 /$ matecconf / 20141102005

(C) Owned by the authors, published by EDP Sciences, 2014

\title{
Comparison between numerical models and CHENSI with experimental data (MUST) within the case of the $0^{\circ}$ approach flow.
}

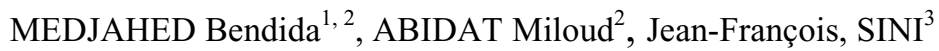 \\ ${ }^{1}$ Laboratoire de Mécanique Appliquée ; Université Des Sciences et de la Technologie d'ORAN, \\ Faculté de Génie Mécanique, BP. 1505, El-Mnaouar, Oran, ALGERIE. \\ ${ }^{2}$ Laboratoire de Modélisation Numérique et Expérimentale des phénomènes Mécanique ; Université \\ Abdelhamid Ibn Badis. Mostaganem, Faculté des Sciences et de la Technologie Mostaganem, \\ ALGERIE. \\ ${ }^{3}$ Laboratoire de Mécanique des Fluides. Equipe Dynamique de l'Atmosphère Habitée; Unité Mixte \\ de Recherche CNRS n ${ }^{\circ}$ 6598, FRANCE.
}

\begin{abstract}
The MUST wind tunnel data set served as a validation case for obstacle-resolving micro-scale models in the COST Action 732 "Quality Assurance and Improvement of Micro-Scale Meteorological Models". The code used for the numerical simulation is code CHENSI, simulations carried out showed a certain degree of agreement between the experimental results and those of the numerical simulation, they highlight the need for proceeding to an experimental campaign but with more measurements and the need for having a good control of determining factors in the exploitation of its results. The aim is to explain the experimental data obtained by atmospheric wind on the physical model. The site company of Mock Urban Setting Test (MUST) was selected to be simulated by the code CEN CHENSI developed by the team of Dynamique of l'atmosphere Habitee of LME/ECN. The code was based on $(\mathrm{K}-\varepsilon)$ model of (Launder and Spalding). For the integration of the PDE (Potential Dimensional equations) constitute the mathematical model, the finite volume method of (Ferziger and Peric) was used within the decade disposition of unknowns MAC of (Harlow and Welck) for the discretisation of PDE terms. The boundary conditions were imposed according to the wall laws (In ground and on buildings) or within Dirichlet condition (Inlet boundary) or of Newman (Outlet boundary or top limit). The numerical domain used was comparable to the one of the atmospheric wind experiences within a three-dimensional Cartesian mesh. Numerical results presented in this study for the mean flow field, turbulent kinetic energy in the direction of wind incidence $0^{\circ}$. For an objective comparison of the CHENSI model performances within other European codes used for MUST configuration simulation. The results obtained by the numerical modelling approach are presented in this paper.
\end{abstract}

Keywords: MUST, COST732 Action, CHENSI Code, $(\mathrm{K}-\varepsilon)$ model.

\section{Introduction}

The aim of the work is to provide data analysis about the use of CHENSI for modeling atmospheric flows, with the help of data obtained in the COST 732 framework (Quality Assurance and Improvement of Micro-scale Meteorological Models) over a mock urban setting (Michael Schatzmann et al 2009). The investigations were carried out with CHENSI, compared to wind tunnel.

The work is relevant for the CHENSI community because it deals with environmental problems, with which CFD and CHENSI might be a good tool to deal. As I can see from the forum it is an interesting topic for several users. It also validates CHENSI data with wind tunnel measurements.
COST is an Intergovernmental European framework for international cooperation between nationally funded research activities. The COST Action 732 (Quality Assurance and Improvement of Micro-scale Meteorological Models) program was carried out to perform proper quality assurance method for micro-scale meteorological models which can model urban pollution dispersion and therefore are used in environmental impact studies and decisions with economic and political consequences. In this framework several CFD codes were evaluated, e.g. FLUENT, MISKAM, Star-CD, CFX.... The first step of the simulations was to determine the wind field and turbulent kinetic energy above a Mock Urban Setting Test which consists of 120 containers as obstacles.

The modelled geometry can be seen in Figure 1. The flow

This is an Open Access article distributed under the terms of the Creative Commons Attribution License 2.0, which permits unrestricted use, distribution, and reproduction in any medium, provided the original work is properly cited. 
enters the domain from the inlet side, perpendicular to the containers longer length. The computational grid used for the CHENSI simulations was taken from the MISKAM and FLUENT simulations to provide an identical environment for comparison. In the wind tunnel measurements 21 reference profiles were measured (Figure 2). The 21 reference profiles are located in the street canyons and behind the buildings, so the fairly undisturbed flow and the wake behind the buildings can also be investigated. Applied in the frame of the MUST exercise of COST732 Action (URL1).

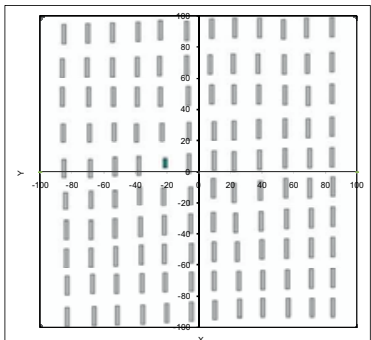

Fig. 1. Modelled geometry.

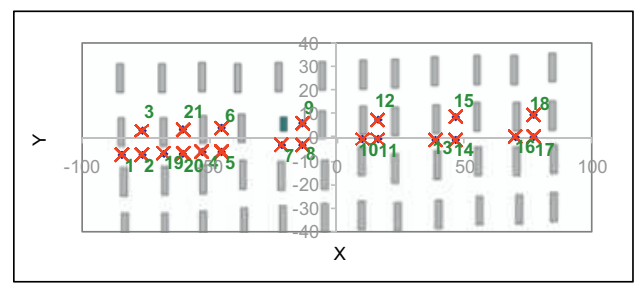

Fig. 2. Velocity profile measurement points

\section{Description of wind tunnel experiments (MUST)}

The wind tunnel data set used in this paper contains flow and dispersion data measured within an idealized urban roughness. The wind tunnel experimental setup originates from the Mock Urban Setting Test (MUST), an extensive field test carried out on a test site of the US Army in the Great Basin Desert in 2001. 120 standard size shipping containers were set up in a nearly regular array of 10 by 12 obstacles (12.2 m long, $2.4 \mathrm{~m}$ wide, and $2.5 \mathrm{~m}$ high) placed in the center of Horizontal Grid along the sides of the roads. The wind tunnel measurements within a scaled model $(1: 75)$ of that configuration were carried out at the University of Hamburg (Bezpalcove, K and F. Harms, 2005). We focus only on main wind direction $\left(0^{\circ}\right)$ which correspond to those cases selected within the COST 732 Action.

\subsection{Set up of model runs}

For the MUST wind tunnel experiment the 120 obstacles of the full scale experiment were modelled on a 1:75 scale and positioned on the wind tunnels turn table as shown in Figure 3. The approach flow conditions in the wind tunnel were adjusted to meet the approach flow measured in full scale. In Figure 4 the measured mean velocity profile and the measured profile for the turbulent kinetic energy are shown. $\mathrm{H}=2.54 \mathrm{~m}$ is the height of the containers in full scale.

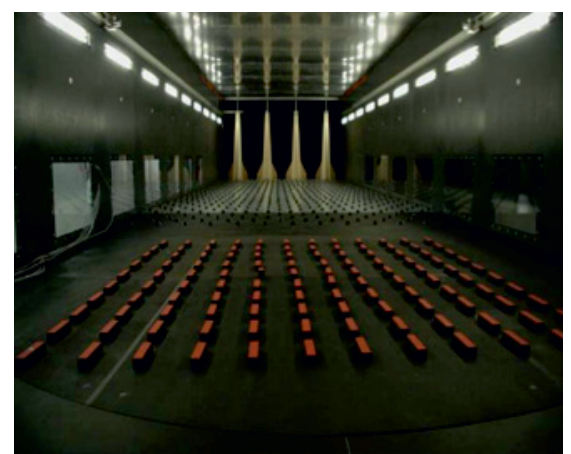

Fig. 3. Setup for the MUST wind tunnel experiment (Bezpalcova and Harms, 2005).

\subsection{The initial conditions and conditions to the limits}

The conditions to the lateral limits are fixed by the direction of wind.

In the case $0^{\circ}$ : On the face of entry (plan (y. z), for which $\mathrm{x}=0$ ) the condition of Dirichlet has been imposed, for the profiles of entry of the middle speed and turbulent kinetic energy. To the level of soil, the classic conditions to the partition have been used, and for the other faces the condition of Neumann has been specified.

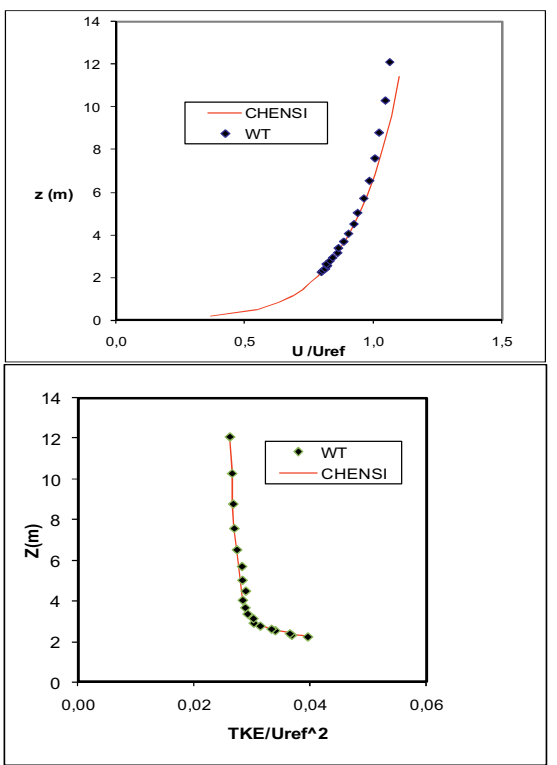

Fig.4. Measured velocity (Top) and turbulent kinetic (Bottom) profile of the approach flow in the wind tunnel.

a)

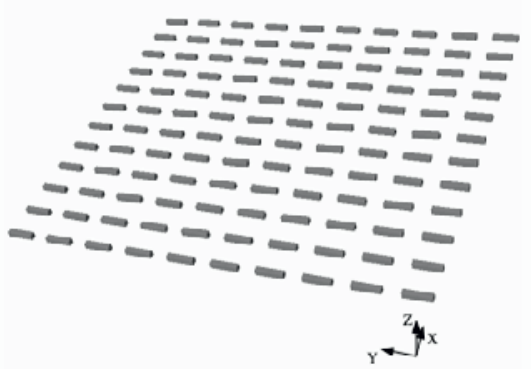


b)

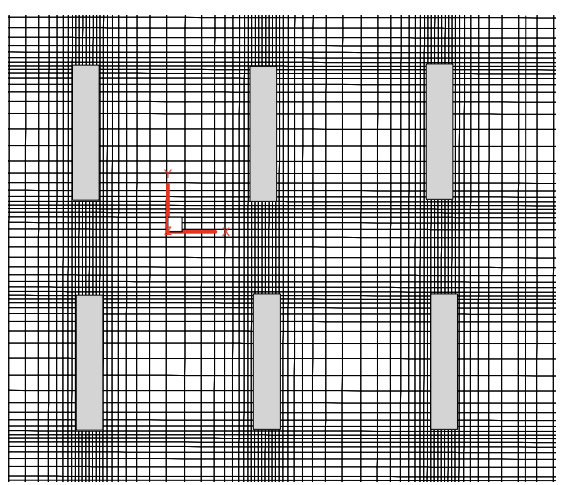

c)

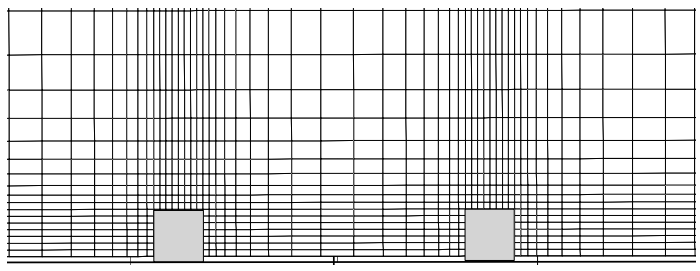

Fig.5.

Domain of simulation a) Three-dimensional domain, b) Vertical cut and c) Horizontal cut (Computational mesh used for numerical simulations).

\section{Models}

The CFD codes applied in this exercise comprise three advanced CFD models, i.e. CHENSI, FLUENT (Fluent, 2006) and MISKAM (Eichhorn, 1989), for the numerical simulation of the three-dimensional flow field and the dispersion of pollutants in the micro-scale. These models employ the widely used 'standard k- $\varepsilon$-model' for the turbulence closure but different implementation of the boundary conditions and different numerical schemes are used. A detailed description of the codes can be found in the model inventory (see link in URL2). All three models used the same domain and grid sizes as well as the same inflow conditions specified by the available experimental data sets.

\section{CFD code validation}

The code CHENSI has been validated for a series of 13 reference flows predicting either of the physical characteristics that are important in the lower urban atmospheric flows: diffusive transport in round and plane jets, and in plumes spreading in a uniform or stratified atmosphere; recirculations in isothermal boundary layers over backward- and forward-facing steps, 2-D and 3-D rectangular blocks; inhomogeneous dynamical and thermal developments in internal plane boundary layers over roughness and temperature steps. These validation tests have been extensively presented and analysed by Sini (1986), Sini and Dekeyser (1987, 1989), Lévi Alvarès et al. (1990), Lévi Alvarès (1991), Zhang (1991) and Mestayer et al (1993). The results show that the standard k- $\varepsilon$ model gives good to excellent predictions for all the mean flow fields and relatively good prediction of the turbulent diffusion, with no systematic deficiencies except in the flow zones where coherent structures, clearly non- isotropic, are of importance in transport processes. In that case (e.g., step or block flows), the k- $\varepsilon$ model significantly under predicts (by about $15-20 \%$ ) the size of recirculating zones. Nevertheless, this first order model can be used for heuristic studies to provide some qualitative ideas on the recirculating flow structure, keeping in mind that some uncertainties can affect the quantitative results.

\section{Results and discussion:}

Three models CHENSI, MISKAM and FLUENT were validated against non-uniform inflow conditions. Before making a direct comparison of the dynamic fields between the wind tunnel measurements and numerical simulations.

\subsection{Component U}

The agreement between observed and modelled uvelocity data upstream of the obstacle was very satisfactory, however, all models agreed excellently with each other and the measurements.

In figure (6), all the models in the towers of 'wide streets'. In agreement with the observations, all codes predicted very accurately the u-velocity of the leeward vortex in the cavity zone behind the obstacle. Finally the profiles of $U$ are almost similar to the wind tunnel measurements, in many cases slightly underestimating them and no remarkable differences can be found between CHENSI and (MISKAM, FLUENT).
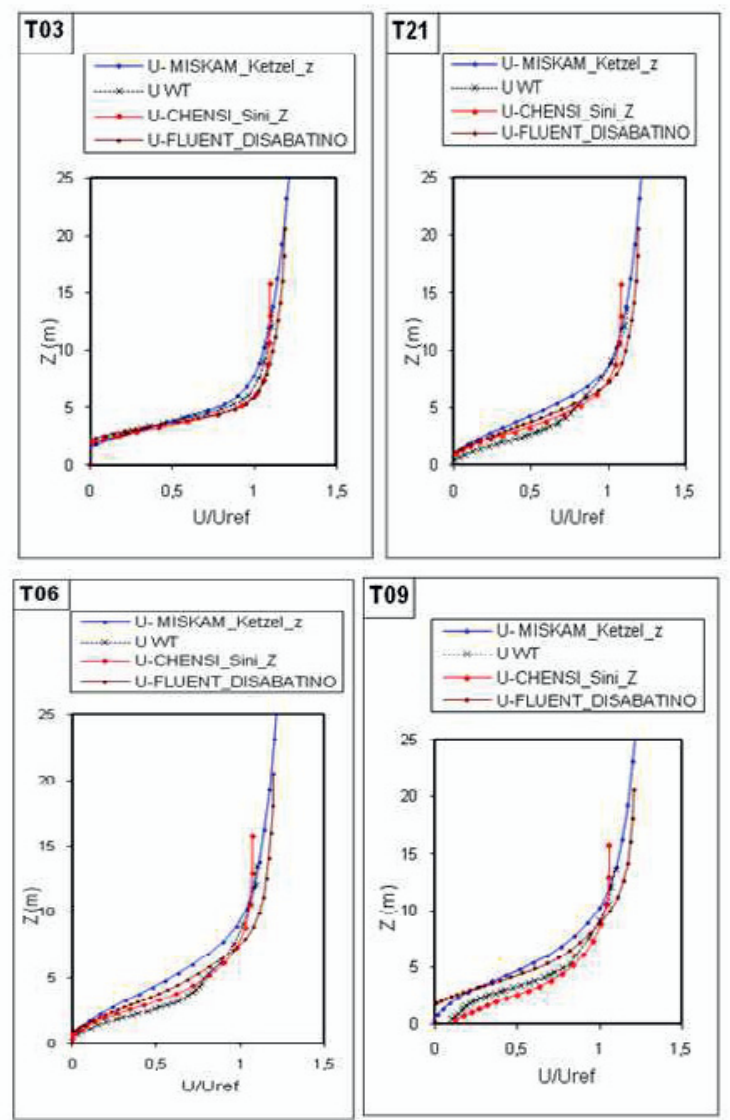


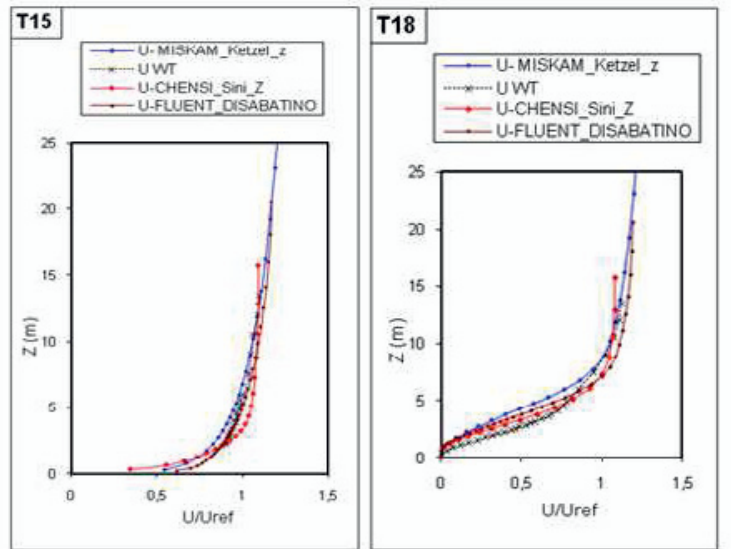

Fig.6. Comparison of experimental and modelled longitudinal velocity $(U)$ profiles normalised with the free stream velocity, are shown at 06 towers, which are representative of 'wide streets' (Tower 3, 6, 9, 15, 18 and 21), cf. Figure 2. The figure is from SavedMetrics_UWtke_UVWtke_0degree_5Dec07.xls with a filter applied (as explained by Olesen and Berkowicz, 2007).

\subsection{Component TKE}

Finally, all of the models had difficulties simulating the turbulent kinetic energy $(\mathrm{k})$ near the up wind face of the obstacles in the three grids are shown at 06 towers, which are representative of 'wide streets' (Tower 3, 6, 9, 12, 15, 18 and 21), cf. Figure 2. In this case, CHENSI came closest to predicting the observed data. However, it is clear that all three models greatly overestimated $\mathrm{k}$ in the impingement region near the up wind obstacle wall, which is a common problem with models using. Further downstream the agreement was good, except that CHENSI over predicted $\mathrm{k}$ just behind the obstacle, while the other three models produced satisfactory results (Fig. 7).

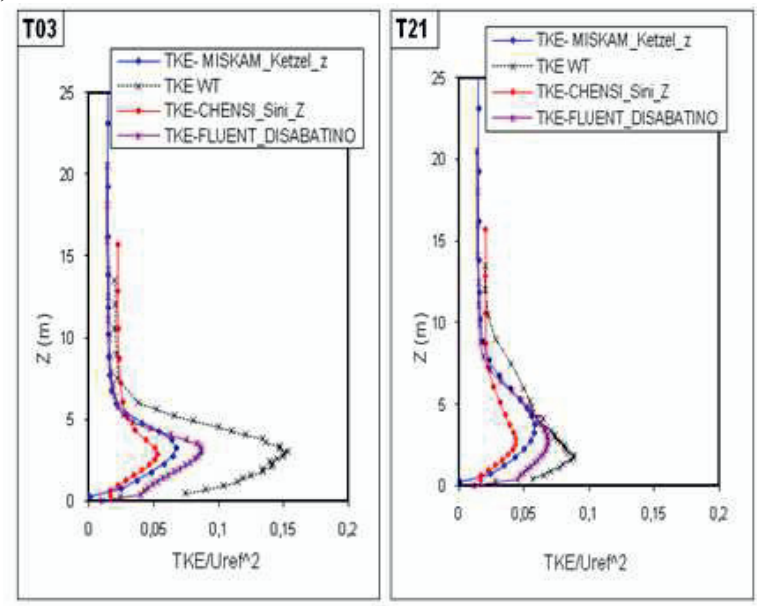

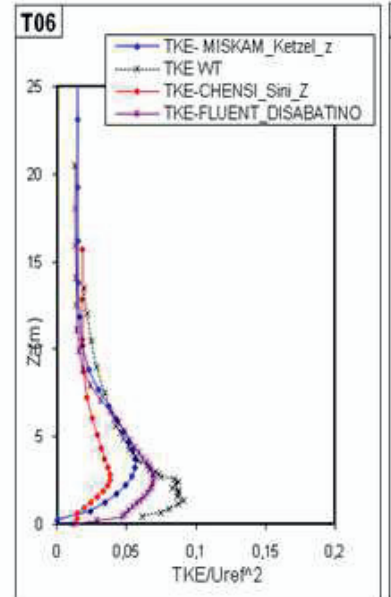
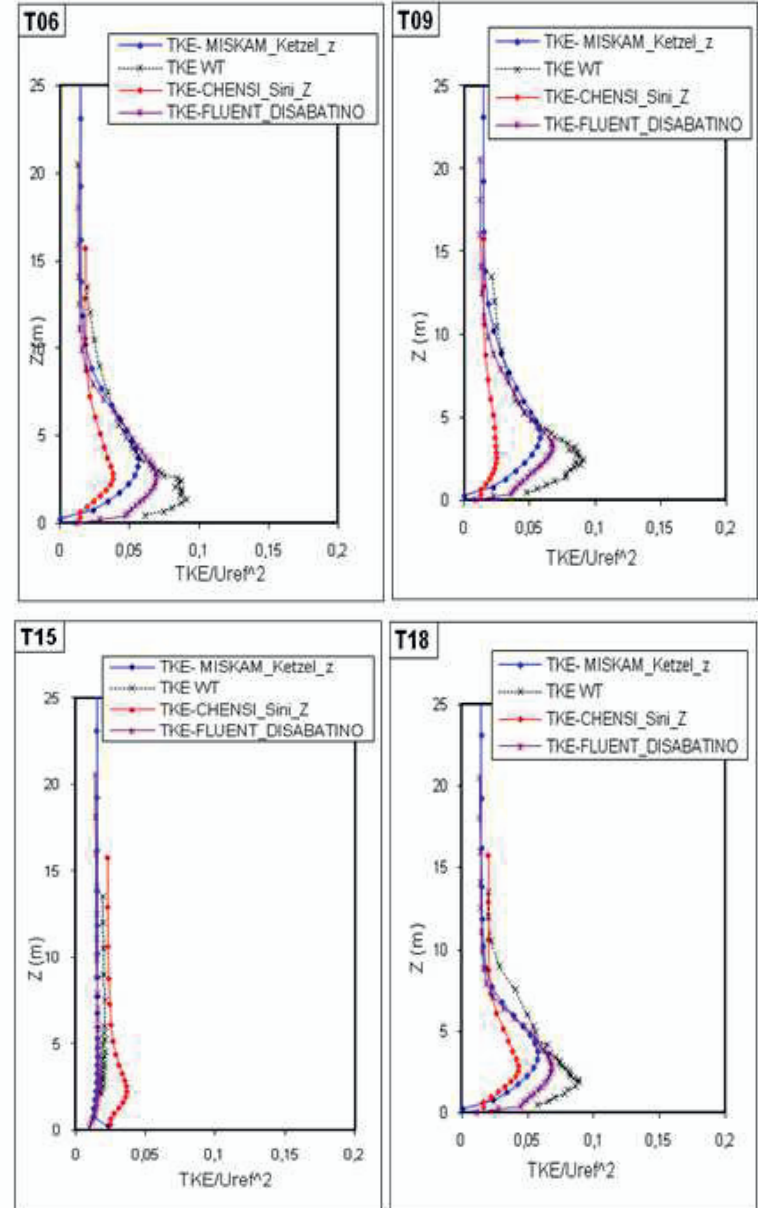

Fig.7. Comparison of experimental and modelled turbulent kinetic energy (k) profiles normalised with the free stream velocity, are shown at 06 towers, which are representative of 'wide streets' (Tower 3, 6, 9, 12, 15, 18 and 21), cf. Figure 2. The figure is from SavedMetrics_UWtke_ UVWtke_0degree_5Dec07.xls with a filter applied (as explained by Olesen and Berkowicz, 2007).

\section{Conclusions}

The present work was carried out within the COST network (creates scientific networks and enables scientists to collaborate in a wide spectrum of activities in research and technology). In order to assure the quality of models for flow in urban and industrial areas, a European COST initiative (see http:/www.cost.esf.org) was launched. COST is an Intergovernmental European framework for international cooperation between nationally funded research activities.

The numerical results were in good agreement with the wind tunnel data, giving a reasonable representation of the general flow pattern. The oncoming flow exhibits an impingement region at the windward side of the obstacle. At the upper leeward edge of the obstacle the flow separates again and leads to an extended lee vortex formed in the cavity zone immediately behind the obstacle which interacts with the horseshoe vortex. The disagreements with the experiment appear close to the reattachment point in the mean longitudinal velocity component and close to the windward obstacle face of turbulent kinetic energy. CHENSI overestimate the 
reattachment length (MISKAM in opposite underestimate) and both models over predict turbulent kinetic energy (TKE) in the impingement region. CHENSI, MISKAM and FLUENT fail to predict the strong gradients of the TKE on the top of the obstacle and close to the lateral obstacle faces. Further model's developer can be another turbulent model implementation and other boundary conditions for the turbulent kinetic energy applying on the obstacle's faces.

\section{Acknowledgements}

The numerical calculations were carried out on the supercomputers of the CNRS national computing centre. Complete computer support was given by the Scientific Council of Institut de Développement et de Recherche pour l'Informatique Scientifique (IDRIS), Orsay, France.

\section{References}

1. Bezpalcova, K. and Harms, F. (2005) EWTL Data Report / Part I: Summarized Test Description Mock Urban Setting Test. Environmental Wind Tunnel Laboratory, Centre for Marine and Atmospheric Research, University of Hamburg.

2. Eichhorn, J. (1989), 'Entwicklung und Anwendung eines dreidimensionalen mikroskaligen Stadtklima-Modells', Dissertation, Universität Mainz, Germany.

3. Ferziger, J. H. \& Peric, M.: 1996, Computational methods for fluid dynamic, Springer-Verlag, New-York - Heidelberg - Berlin.

4. FLUENT (2006): FLUENT V6.3 User's guide. Lebanon, New Hampshire: Fluent Inc.

5. Hanjàlic R. and Launder B. E. (1972) A Reynolds stress model of turbulence and its application to thin shear flows. J. Fluid Mech. 52, 609-638.

6. Harlow, F. H \& Welch, J. E.: 1965, Numerical calculation of time-dependent viscous incompressible flow of fluid with free surface, Phys. Fluids 8, 2182-2182.

7. Launder B. E. and Spalding D. B. (1974) The numerical computation of turbulent flows. Comput. Meth. Appl. Mech. Engng 3, 269-289.

8. Launder B. E., Reynolds W. C., Rodi W., Mathieu J. and Jeandel D. (1984) Turbulence Models and their Applications. Collection de la Direction des Etudes et Recherches d'Electricité de France, 2, No, 56, Eyrolles Ed., Paris.

9. Lévi Alvarès S. (now Anquetin S.), Sini J.-F., Zhang C. X. and Sacré C. (1990) Tests for a flow simulation around a isolated block, $\mathrm{k}$ e. turbulence model improvements. AMS 9th Symp. on Turbulence and Diffusion, Roskilde,
Denmark, May 1990, A.M.S., Boston, Massachusetts, pp. 145-147.

10. Lévi Alvarès S. (now Anquetin S.) (1991) Simulation numérique des écoulements urbains fi l'échelle d'une rue fi l'aide d'un module $k$ - $e$ (in French), Thèse de doctorat, Ecole Centrale de Nantes, 1 rue de la Noë, 44072 Nantes Cedex, France.

11. Mestayer P. G., Sini J. F., Rey C., Anquetin S., Badri Kusuma S. M., Lakehal D. and Moulinec Ch. (1993) Pollutant dispersion in the urban atmosphere: simulation of turbulent flows using a k $\varepsilon$ model. ERCOFTAC Bull. 16, 2228.

12. Michael Schatzmann, Helge Olesen and Jörg Franke (2009):' COST 732 Model Evaluation Case Studies', COST Action 732, Quality Assurance and Improvement of Microscale Meteorological Models, 1 June 2009.

13. Olesen, H.R. and Berkowicz, R. (2007): Guide to Excel sheets for MUST experiment http://www2.dmu.dk/atmosphericenvironment/D ocs/SpreadsheetInfo.pdf

14. Sini J.-F. (1986) Modé1isation d'6coulements turbulents libres bidimensionnels avec effets de flottabilité -- Cas du panache en milieu stratifié. (in French), Thèse de doctorat,Université d'AixMarseille II, IMST, 12, Avenue du Général Leclerc, 13003-Marseille, France.

15. Sini J. F. and Dekeyser I. (1987) Numerical prediction of turbulent plane jets and forced plumes by use of the k- $\varepsilon$; model of turbulence. Int. J. Heat Mass Transfer 30,1787 1801.

16. Sini J. F. and Dekeyser I. (1989) Numerical prediction of turbulent plane buoyant jets discharging in a stratified stagnant or flowing ocean. Numer. Heat Transfer 16,371-387.

17. Zhang C.-X. (1991) Simulation numérique d'écoulements turbulents autour d'un obstacle. (in French), Thèse de doctorat, Ecole Centrale de Nantes, 1 rue de la Noê, 44072 Nantes Cedex, France.

18. URL1: The MUST exercise of COST732 Action URL http://www.mi.uni- hamburg.de/cost732

19. URL2: CFD working group URL http://www.dmu.dk/AtmosphericEnvironment/tr apos/cfd-wg.htm 